Case Study

\title{
lower limbs kinematic assessment of the effect of a gym and hydrotherapy rehabilitation protocol after knee megaprosthesis: a case report
}

\author{
Nicola Lovecchio ${ }^{1)}$, Luciana Sciumè ${ }^{2)}$, Matteo Zago ${ }^{1)}$, Lorenzo Panella ${ }^{3)}$, \\ Maurizio Lopresti ${ }^{3)}$, Chiarella SForza ${ }^{1)^{*}}$ \\ 1) Laboratorio di Anatomia Funzionale dell'Apparato Locomotore, Functional Anatomy Research \\ Center (FARC), Department of Biomedical Sciences for Health, Università degli Studi di Milano: \\ via Mangiagalli 31 I-20133 Milano, Italy \\ 2) Scuola di Specializzazione in Medicina Fisica e Riabilitazione, Università degli Studi di Milano, Italy \\ 3) S.C. Medicina Fisica e Riabilitazione, Istituto Ortopedico G. Pini, Italy
}

\begin{abstract}
Purpose] To quantitatively assess the effect of a personalized rehabilitation protocol after knee megaprosthesis. [Subject and Methods] The gait patterns of a 33-year-old male patient with knee synovial sarcoma were assessed by a computerized analysis before and after 40 rehabilitation sessions. [Results] The rehabilitation protocol improved the gait pattern. After rehabilitation, hip flexion was nearly symmetric, with normalized affected limb hip flexion, and improved ankle flexion. Ankle in/eversion was asymmetric and did not improve after physiotherapy. Before physiotherapy, the hip flexion on the affected side anticipated the movement but nearly normalized in the follow-up assessment. Hip abduction range of motion increased, with wider movements and good balance. Knee range of motion nearly symmetrized, but maintained an anticipated behavior, without shock absorption at heelstrike. [Conclusion] Instrumental gait analysis allowed us to gain evidence about the training and how to expand rehabilitative interventions to improve efficacy. In particular, we recommend quadriceps and gastrocnemius eccentric contraction training (to improve the shock absorption phase, preventing early failures of the prosthesis); one-leg standing performance (to improve the support phase of the affected limb); adductor strength training (to aid in hip control during the swing phase); and peroneus strength training (to increase ankle joint stabilization). Key words: Knee megaprosthesis, Gait, Limb-saving surgery
\end{abstract}

(This article was submitted Nov. 10, 2015, and was accepted Dec. 12, 2015)

\section{INTRODUCTION}

Synovial sarcoma is a rare soft tissue malignancy with an estimated incidence of 2.75 per 100,000. It is most common in the third-to-fifth decades of life and involves the extremities and, in particular, the lower limbs ${ }^{1-3)}$. The classic treatment of sarcomas of the extremity is radical resection or amputation ${ }^{4)}$. Currently, a more conservative attitude predominates using wide or marginal tumor resection and reconstruction with modular or custom-made endoprostheses. Since most of these patients are young, long-term functional results are critical ${ }^{5-7}$. These patients can achieve full independence after rehabilitation. However, the best rehabilitation technique remains conjectural, and its actual guidelines are undocumented ${ }^{8,9)}$. Shehadeh et al. ${ }^{8)}$ conducted a pilot study on a rehabilitation protocol addressing the five major anatomical regions encountered in limb salvage surgery, including timeline (ranging from postoperative day one to six months), detailing specific exercises, restrictions and goals to achieve, but did not report an objective quantitative evaluation.

The most common daily activity is walking, and the gait pattern of these patients is often different from normalcy ${ }^{10)}$, with

*Corresponding author. Chiarella Sforza (E-mail: Chiarella.sforza@unimi.it)

(C2016 The Society of Physical Therapy Science. Published by IPEC Inc.

This is an open-access article distributed under the terms of the Creative Commons Attribution Non-Commercial No Derivatives (by-nc-nd) License $<$ http://creativecommons.org/licenses/by-nc-nd/4.0/>. 
Table 1. History of surgery undergone by the patient

\begin{tabular}{lll}
\hline \multicolumn{2}{c}{$\begin{array}{c}\text { Follow } \\
\text { up } \\
\text { (month) }\end{array}$} & \multicolumn{1}{c}{ Type of surgery } \\
\hline 1 & 0 & Knee total resection and reconstruction with distal femur megaprosthesis and tibial allograft-prosthesis composite. \\
2 & 1 & Surgical wound revision and suturing of the patellar tendon. \\
3 & 6 & $\begin{array}{l}\text { Transposition of medial gastrocnemius muscle flap, transposition of semitendinosus and gracilis tendons, patella-tibia } \\
\text { cerclage. }\end{array}$ \\
4 & 39 & $\begin{array}{l}\text { Revision: polyethylene components replacement and revision of the extensor mechanism. } \\
5\end{array}$ \\
63 & $\begin{array}{l}\text { Revision: tibial allograft removal, tibial/distal femur prosthesis revision and a coating of Trevira tube over the tibial } \\
\text { prosthesis. }\end{array}$ \\
\hline
\end{tabular}

a lower preferred speed, a longer step length of the non-operated limb and a lengthened stride time ${ }^{7,11)}$. Moreover, during the stance phase, two major altered gait patterns are described: reduced knee flexion during loading response (stiff knee gait) and reduced knee extension in the late stance phase (flexed knee gait) ${ }^{5,7,12)}$. It is unknown whether these abnormal patterns lead to secondary musculoskeletal impairments. A stiff or hyperextended knee gait may reduce the survival of the prosthesis, and rehabilitation should focus on restoring a more natural gait pattern ${ }^{5,13,14)}$. Colangeli et al. ${ }^{13)}$ investigated kinematic and kinetic gait parameters after surgery, and compared total knee replacements (TKR) versus osteochondral allograft (AL) relative to healthy control subjects. The stance duration seemed comparable to the control group in both surgical protocols, even when TKR patients showed a hyperextension pattern during loading response while AL patients showed it only during heel strike. Moreover, the EMG signal indicated a reduced activity of the rectus femoris in TKR patients, showing that knee stability was performed using the mechanical structure of the prosthesis.

In the current case report, gait patterns were longitudinally assessed in a patient with synovial sarcoma of the knee who underwent resection and reconstruction with megaprosthesis subjected to multiple revisions. In particular, we investigated whether there were biomechanical deficits during stance or gait ${ }^{5,10-12,14)}$. In a previous pilot study, we investigated a single exercise (squat) to estimate the effect of rehabilitation ${ }^{15)}$; in the current report, we expanded the assessment to a daily activity.

Our primary aim is to evaluate if a rehabilitation protocol that combines gym and hydrotherapy exercises is effective in recovering the normal gait pattern in a short-term perspective. The secondary aim is to understand which kind of exercise should be included to enhance the rehabilitation process. Our report describes the details of an integrated rehabilitation process involving water activities that could modify the characteristics of a 'stiff/hyperextended knee gait' to reduce the endoprosthesis overload.

\section{SUBJECT AND METHODS}

The patient was a 28-year-old man at the time of the synovial sarcoma diagnosis (monophasic fibrous, right knee). He was a pharmacist, and worked in the standing position, maintaining an erect posture or walking for about eight hours a day. As described by Lovecchio et al. ${ }^{15)}$, he underwent intralesional surgery and subsequent knee total resection and reconstruction with distal femur megaprosthesis and tibial allograft-prosthesis composite (first surgery, Table 1) ${ }^{16,17)}$. Afterwards, he had other surgical treatments for mechanical failure of his prosthesis. After the fifth surgery, the patient was locked with the knee brace in extension for 30 days and then kept the unlocked brace an additional 30 days. Considering his unique clinical history, we decided to project a novel rehabilitation program and to perform a computerized analysis of his gait patterns to help understand the anatomic and biomechanical reasons underlying the multiple, frequent revisions of knee megaprosthesis.

The first evaluation took place one month after the fifth surgery ${ }^{15)}$. We noted an adherent scar on the medial aspect of his right thigh and measured the passive range of motion (RoM) on his lower limbs. The patient had a reduced knee flexion $\left(100^{\circ}\right)$ and an increased extension $\left(0^{\circ} \text { to } 10^{\circ}\right)^{18)}$ on his right side compared to the contralateral side. The strength of knee extension was $3 / 5$ as measured by manual muscle testing according to the British Medical Research Council scale (BMRC). No other strength deficit was identifiable in his right lower limb $\left.{ }^{19}, 20\right)$. While standing, he showed right side genu recurvatum. At that time, he wore an unlocked knee brace and began to walk with full-weight bearing as tolerated on the right leg.

We performed data collection and analysis procedures with a motion analysis system (BTS, Milano, Italy). Nine infrared cameras recorded at $120 \mathrm{~Hz}$ the 3D coordinates of 25 passive reflective markers placed on the patient's skin as described by Lovecchio et $\mathrm{al}^{15}{ }^{\text {) }}$. All procedures were in accordance with the Declaration of Helsinki, and were preventively approved by the hospital ethic committee. Once we obtained written informed consent, the patient was tested barefooted and wearing a bathing suit. He walked along a six-meter corridor at a self-chosen speed, looking straight ahead, with no movement restrictions imposed. We did not provide a crutch, handrail or therapist assistance. The patient never reported discomfort during or after the completion of a minimum of 20 walking stride cycles. We repeated the procedure before and after 40 physical therapy sessions (three months later). 
Table 2. The patient progressed from 10 to 30-40 repetitions of each exercise. Exercise load was based on the patient's level of perceived exertion

\begin{tabular}{|c|c|c|c|c|}
\hline \multicolumn{5}{|l|}{ Rehabilitation program } \\
\hline \multirow{4}{*}{ Gym session } & $\begin{array}{l}\text { Isometric } \\
\text { contraction }(5 \mathrm{~s})\end{array}$ & $\begin{array}{l}\text { Quadriceps, hamstring and } \\
\text { gluteus }\end{array}$ & & \\
\hline & Co-contraction & knee at $0^{\circ}, 30^{\circ}$ and $90^{\circ}$ of flexion & & \\
\hline & Mobilization & $\begin{array}{l}\text { Active-assisted and active } \\
\text { hip-knee-ankle joint mobilization }\end{array}$ & $\begin{array}{l}\text { Knee flexion stopping the } \\
\text { movement at } 15^{\circ}, 30^{\circ}, 60^{\circ} \text { and } 90^{\circ}\end{array}$ & $\begin{array}{l}\text { Squat exercises at } \\
\text { self-chosen depth }\end{array}$ \\
\hline & Standing trials & One-legged standing & $\begin{array}{l}\text { Standing with eyes open/closed on } \\
\text { firm surface/foam cushion }\end{array}$ & $\begin{array}{l}\text { Gait training on flat } \\
\text { surfaces and on stairs }\end{array}$ \\
\hline \multirow{2}{*}{$\begin{array}{l}\text { Hydrokinesiotherapy } \\
\text { (water level, } 1.20 \mathrm{~m} \text { ) }\end{array}$} & $\begin{array}{l}\text { Lower limb } \\
\text { exercise }\end{array}$ & $\begin{array}{l}\text { Walking forward, backward and } \\
\text { sideways }\end{array}$ & Skipping exercises & Half-squat \\
\hline & Balance exercise & Single leg balance & Keeping a board under the foot & \\
\hline
\end{tabular}

The global coordinates system was defined as follows: X-axis, anteroposterior direction, positive forward; Y-axis, craniocaudal, pointing upwards; Z-axis, orthogonal to $\mathrm{X}$ and $\mathrm{Y}$, pointing to the right. We determined the heel-strike events by inspecting malleoli marker trajectories and 3-D body reconstruction. For each cycle, single, double support and swing phase durations were calculated. Subsequently, the gait cycles were time-normalized. We computed step width as the distance on the transverse plane between the positions of the center of mass of each foot during the stance phase of consecutive steps. The RoM of hip and knee flexion/extension, hip abduction/adduction, pelvis rotation, inclination, and tilt, were calculated. All joint angles were estimated computing the rotation matrix between contiguous anatomical body segments (Cardan ZY' $\mathrm{X}^{\prime}$ ' convention). We evaluated bilateral asymmetries through the Symmetry Angle (SA) between group medians ${ }^{21)}$. SA is a robust symmetry index computed as $100^{*}\left[45^{\circ}-\arctan \left(\mathrm{X}_{\text {left }} / \mathrm{X}_{\text {right }}\right)\right] / 90^{\circ}$, where $\mathrm{X}_{\text {left/right }}$ are the left and right values. It ranges between $0 \%$ (perfect symmetry) and $100 \%$ (equal and opposite values).

We computed descriptive statistics (median and 95\% Confidence Intervals for non-normal populations, CI) separately for pre and post rehabilitation assessments of the affected (AL) and non-affected limbs (NAL) ${ }^{22}$.

Over an eight-week period, the patient underwent 40 physical therapy sessions of approximately 90 minutes each. A session included an initial thigh scar massage (performed by a therapist), and gym and hydrotherapy programs, each lasting 45 minutes, where the patient was encouraged to complete three sets of ten repetitions of each exercise unless prevented by fatigue or pain (Table 2). The correctness of execution, stability in balance, a general motor control and improvements in strength were also considered key factors to progress in the rehabilitation process. During rehabilitation, the patient's compliance was always high with total physical and mental participation; no injuries or illness occurred to modify the plan of the process.

\section{RESULTS}

We completed a follow-up gait evaluation at the end of rehabilitation; we did not detect disability by the FIM (126/126) and the patient returned to his work. We did not observe any major modifications in his affected knee passive RoM. The strength of knee extension increased from three to four according to the BMRC, and genu recurvatum on standing decreased. The patient was walking without a crutch and knee brace. The spatiotemporal parameters of his gait cycle remained substantially unchanged between measurements (Table 3). In particular stance percentage increased in post rehabilitation (the SA increased), while duration, cadence, and step length remained, practically, equal. Step width reduced its CI. Hip flexion decreased while ab/adduction revealed an improvement; both improved in symmetry (Table 4). Hip rotation decreased its CI in both sides with different magnitudes (SA increased from 1.5 to 4.3\%). The AL knee RoM reduced its median value and variability (CI). Ankle in/eversion showed a general improvement in symmetry while flexion was more asymmetric, even with a gain in AL RoM. On the AL, maximal hip flexion angles were lower than those measured on the NAL (Fig. 1). In the transverse plane, the patient externally rotated his AL hip before $50 \%$ of stride, with a reduced movement during heel contact and swing phases (Fig. 2). On the NAL, hip external rotation increased during loading response (Fig. 2, from 0 to $50 \%$ of gait cycle). The above-mentioned gait asymmetry also appeared in the kinematic parameters of the knee. Indeed, flexion was greater in the AL both pre and post rehabilitation. Angular displacement showed a continuous knee hyperextension during loading response and mid-stance (from 0 to $35 \%$ of gait cycle). At toe-off, the affected knee reached greater and earlier peak flexion (Fig. 3). A reduction in right knee hyperextension was clearly noticeable during the stance phase (Fig. 3). Knee flexion RoM decreased on both sides after rehabilitation. During the swing phase, knee flexion of the AL decreased becoming more similar to the contralateral value (Fig. 3), while right ankle dorsiflexion increased. 
Table 3. Median and 95\% ICs of spatio-temporal data for the patient with multiple revisions of knee megaprosthesis during unassisted gait, 1 and 4 months after his fifth surgery

\begin{tabular}{|c|c|c|c|c|c|c|c|}
\hline \multirow{2}{*}{ Parameter (deg) } & \multirow{2}{*}{ Limb } & \multicolumn{2}{|c|}{ Pre rehabilitation } & \multirow{2}{*}{ SA $(\%)$} & \multicolumn{2}{|c|}{ Post rehabilitation } & \multirow{2}{*}{ SA $(\%)$} \\
\hline & & median & $\mathrm{ICs}$ & & median & ICs & \\
\hline \multirow[t]{2}{*}{$\%$ stance } & $\mathrm{AL}$ & 48.5 & $46.6-50.4$ & \multirow{2}{*}{2.2} & 47.5 & $44.8-48.9$ & \multirow{2}{*}{3.0} \\
\hline & NAL & 52.0 & $50.7-53.3$ & & 53.0 & $51.1-54.9$ & \\
\hline \multirow[t]{2}{*}{$\%$ swing } & $\mathrm{AL}$ & 51.5 & $49.6-53.4$ & \multirow{2}{*}{2.2} & 52.5 & $49.8-55.2$ & \multirow{2}{*}{3.0} \\
\hline & NAL & 48.0 & $46.7-49.3$ & & 47.0 & $45.1-48.9$ & \\
\hline \multirow[t]{2}{*}{ Cycle duration (s) } & $\mathrm{AL}$ & 1.32 & $1.27-1.36$ & \multirow{2}{*}{0.0} & 1.31 & $1.29-1.34$ & \multirow{2}{*}{0.2} \\
\hline & NAL & 1.32 & $1.28-1.37$ & & 1.31 & $1.29-1.32$ & \\
\hline \multirow[t]{2}{*}{ Cadence (step/s) } & $\mathrm{AL}$ & 0.76 & $0.74-0.78$ & \multirow{2}{*}{0.0} & 0.76 & $0.74-0.77$ & \multirow{2}{*}{0.2} \\
\hline & NAL & 0.76 & $0.73-0.79$ & & 0.76 & $0.76-0.77$ & \\
\hline \multirow[t]{2}{*}{ Step length (m) } & $\mathrm{AL}$ & 1.14 & $1.04-1.24$ & \multirow{2}{*}{0.0} & 1.16 & $1.13-1.34$ & \multirow{2}{*}{0.5} \\
\hline & NAL & 1.14 & $1.11-1.16$ & & 1.16 & $1.14-1.19$ & \\
\hline Step width (m) & & 0.08 & $0.02-0.15$ & & 0.08 & $0.06-0.10$ & \\
\hline
\end{tabular}

SA: symmetry angle (based on group medians); AL/NAL: affected/non affected limb

Table 4. Median and 95\% ICs of kinematic parameters for the patient with multiple revisions of knee megaprosthesis during unassisted gait 1 and 4 months after his fifth surgery

\begin{tabular}{|c|c|c|c|c|c|c|c|}
\hline \multirow{2}{*}{ Parameter (deg) } & \multirow{2}{*}{ Limb } & \multicolumn{2}{|c|}{ Pre rehabilitation } & \multirow{2}{*}{ SA $(\%)$} & \multicolumn{2}{|c|}{ Post rehabilitation } & \multirow{2}{*}{ SA $(\%)$} \\
\hline & & median & ICs & & median & ICs & \\
\hline \multirow[t]{2}{*}{ Hip flexion } & $\mathrm{AL}$ & 44.3 & $43.6-45.0$ & \multirow{2}{*}{2.1} & 43.8 & $42.9-44.7$ & \multirow{2}{*}{1.4} \\
\hline & NAL & 50.2 & $40.8-52.4$ & & 42.0 & $39.9-44.1$ & \\
\hline \multirow[t]{2}{*}{ Hip ab/adduction } & $\mathrm{AL}$ & 19.9 & $18.6-21.2$ & \multirow{2}{*}{9.9} & 21.0 & $20.1-22.0$ & \multirow{2}{*}{7.2} \\
\hline & NAL & 27.3 & $25.9-28.7$ & & 26.4 & $25.0-27.9$ & \\
\hline \multirow[t]{2}{*}{ Hip rotation } & $\mathrm{AL}$ & 10.3 & $9.7-10.9$ & \multirow{2}{*}{1.5} & 9.5 & $9.1-9.9$ & \multirow{2}{*}{4.3} \\
\hline & NAL & 10.8 & $9.2-12.4$ & & 10.6 & $9.8-11.9$ & \\
\hline \multirow[t]{2}{*}{ Knee flexion } & $\mathrm{AL}$ & 71.1 & $70.3-71.9$ & \multirow{2}{*}{10.9} & 66.7 & $66.3-67.6$ & \multirow{2}{*}{9.9} \\
\hline & NAL & 50.2 & $48.0-52.4$ & & 48.8 & $46.6-51.0$ & \\
\hline \multirow[t]{2}{*}{ Ankle in/eversion } & $\mathrm{AL}$ & 35.0 & $32.8-35.6$ & \multirow{2}{*}{9.2} & 34.6 & $31.8-37.4$ & \multirow{2}{*}{5.8} \\
\hline & NAL & 35.0 & $32.9-37.1$ & & 41.5 & $37.2-45.9$ & \\
\hline \multirow[t]{2}{*}{ Ankle flexion } & $\mathrm{AL}$ & 25.0 & $23.7-28.7$ & \multirow{2}{*}{4.0} & 27.3 & $25.1-29.6$ & \multirow{2}{*}{6.1} \\
\hline & NAL & 33.5 & $31.3-35.6$ & & 33.2 & $24.1-42.2$ & \\
\hline
\end{tabular}

SA: symmetry angle (based on group medians). Values are average peak values for the considered parameter; AL/NAL: affected/non affected limb

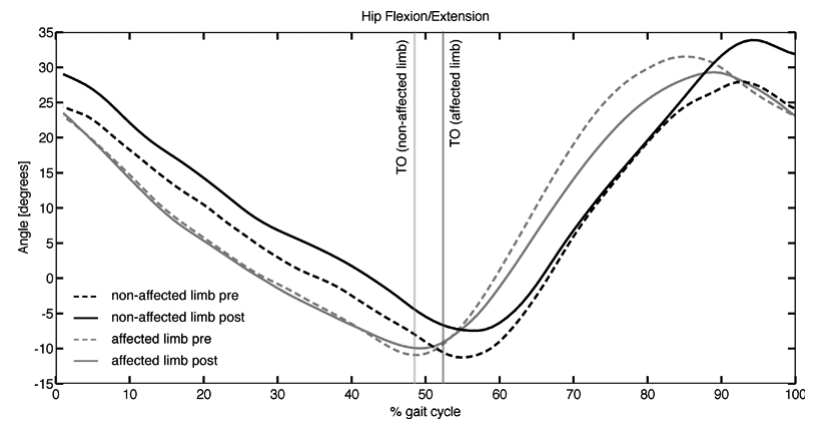

Fig. 1. Hip flexion/extension angle during the gait cycle for the affected (gray) and non-affected (black) limb, in both the pre- (dashed) and post-intervention (solid line) measurements. Vertical lines indicate the average toe-off instant

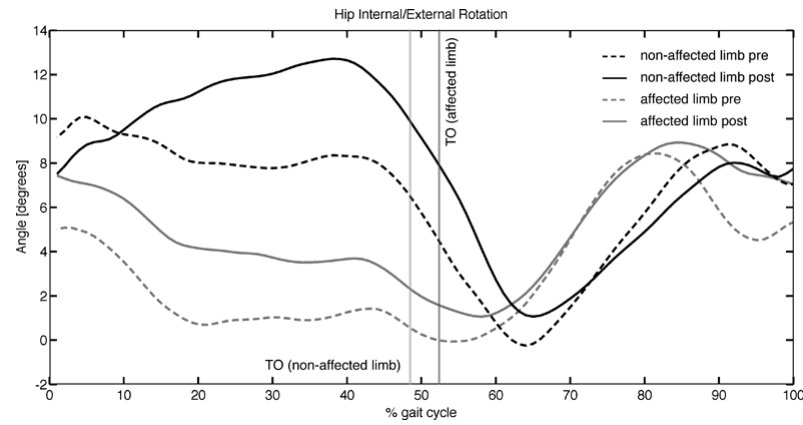

Fig. 2. Hip internal/external rotation angle during the gait cycle for the affected (gray) and non-affected (black) limb, in both the pre- (dashed) and post-intervention (solid line) measurements. Vertical lines indicate the average toe-off instant 


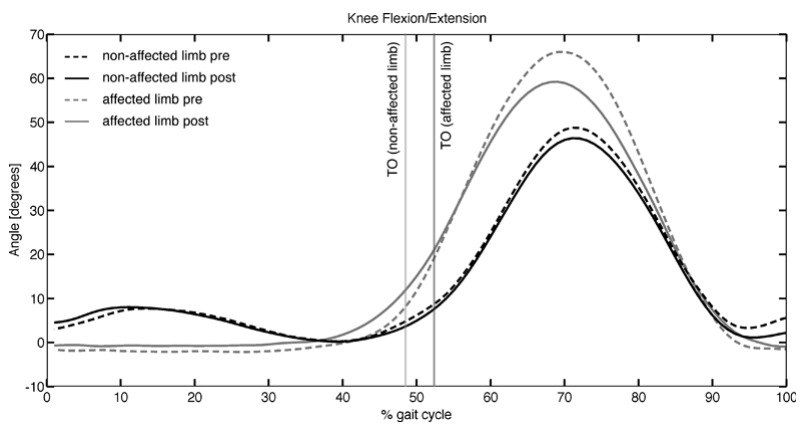

Fig. 3. Knee flexion/extension angle during the gait cycle for the affected (gray) and non-affected (black) limb, in both the pre- (line) and post-intervention (solid line) measurements. Vertical lines indicate the average toe-off instant.

\section{DISCUSSION}

Advanced surgical techniques and endoprostheses manufacturing technologies allow for new conservative solutions of body impairments, as in the case of megaprostheses for limb tumor surgery ${ }^{4,5}$, 7). At the same time, a critical issue is the process of rehabilitation where a long-term outcome becomes crucial for the general wellbeing of the patients. Thus, specific indications about knee movements, for instance, hyperextension during loading response or knee flexion-extension during mid-stance, are critical. Objective measures are necessary to reduce rehabilitation process length, obtain an efficient intervention and reduce mechanical failure that may lead to the early revision of prostheses ${ }^{11)}$. One study reports a median prosthetic survival of 130 months for distal femoral resections and 117 months for proximal tibial resections ${ }^{23)}$, longer than those found in the present patient.

Thus, we evaluated gait parameters and lower limb RoM before and after a specific rehabilitation protocol to define clinical indications based on objective quantitative data ${ }^{15}$. Our rehabilitation protocol, combining gym, and hydrokinesis produced improvements in gait pattern: cycle duration and cadence remained constant while step length improved and step width decreased. Moreover, more functional ankle RoM and hip flexion, as previously reported in literature ${ }^{12)}$, were obtained. The patient reached symmetry in knee and hip RoM (except for rotation) as well. In particular, step width indicates a gain in stability ${ }^{24)}$ while the CIs of step length remained mostly overlapped. The stance phase result was faster in the AL, with a shortened duration relative to normalcy ${ }^{25)}$. We suggest increasing exercises based on one-leg standing, thus improving the support phase of the AL.

After rehabilitation, hip flexion showed similar and symmetrical RoM in both limbs (overlapped CIs, Table 4) without flexion compensation in the contralateral hip ${ }^{7,12)}$. The slight reduction in hip flexion of the AL reported in our study is similar to a previously reported case of knee reconstruction using a hingeless prosthesis ${ }^{12)}$. The reduction in hip flexion may be associated with an improvement in ankle flexion as an efficient and functional pattern to allow toe clearance (at least 10 degrees in dorsiflexion). Indeed, although the AL did not show large increments in ankle flexion (Table 4), a positive trend was found, in accordance with previous reports ${ }^{7}$. Ankle in/eversion showed a reduced asymmetry (lower SA), suggesting that rehabilitation plays a role in ankle joint stabilization and distal control ${ }^{24)}$.

Regarding hip flexion (Fig. 1), we noticed an unusual performance: the healthy limb started to flex after the end of the toe-off phase while it anticipated hip flexion on the contralateral side. In our opinion, this is a pattern strategy to facilitate the toe-off. After rehabilitation, hip flexion of the AL was delayed and neared the healthy side RoM. Affected hip abduction increased its RoM: the wider movement may be due to a restored self-confidence ${ }^{26)}$. Further, this condition could reveal a good balance during single support, allowing larger movements during the swing phase. This is a positive effect of treatment that should be selectively trained favoring adductor muscles contraction ${ }^{27)}$. After rehabilitation, hip internal rotation increased (Fig. 2, 10-40\% of gait cycle): this may be the result of a major load acceptance capability. Additionally, the AL hip RoM (flexion and ab/adduction) became close to the healthy side, showing a normal kinematic path ${ }^{28)}$.

Knee movement patterns on the AL revealed an improvement after the toe-off but maintained an anticipated behavior. That is, the toe-off occurred with an excessively flexed knee (Fig. 3, 40-50\% of gait cycle) that reduced the power lift ${ }^{24)}$.

Similar to previous studies ${ }^{9,14}$, after the intervention, the loading response (0-15\% of gait cycle) decreased ${ }^{10}$ and still occurred without knee flexion (damping action). Because of this gait pattern, there was no shock absorption at the beginning of the heel-strike. We visualized shock absorption at the initial stance phase in the contralateral knee as a small increase in knee flexion (loading response); this finding is consistent with the literature at long-term follow-up ${ }^{5)}$. To prevent early failures of the prosthesis ${ }^{12}$, we suggest additional exercises for eccentric quadriceps/gastrocnemius contraction, performed within the first three postoperative months ${ }^{29)}$.

In summary, instrumental gait analysis before and after a specific rehabilitation process provides evidence about the 
proposed exercise training, suggesting further rehabilitative interventions to improve effectiveness. In particular, we recommend quadriceps and gastrocnemius eccentric contraction training, one-leg standing performance, and adductor and peroneus strength training. In conclusion, this functional analysis could define a new approach to specific rehabilitation protocols for selected patients.

\section{REFERENCES}

1) Deshmukh R, Mankin HJ, Singer S: Synovial sarcoma: the importance of size and location for survival. Clin Orthop Relat Res, 2004, (419): 155-161. [Medline] [CrossRef]

2) Moatasim A, U1 Haque A: Monophasic synovial sarcoma diagnosed on FNAC. Int J Pathol, 2004, 2: 108-110.

3) Friedman MV, Kyriakos M, Matava MJ, et al.: Intra-articular synovial sarcoma. Skeletal Radiol, 2013, 42: 859-867. [Medline] [CrossRef]

4) Papadakis SA, Bisbinas I, Mitsitsikas T, et al.: Synovial sarcoma arising in the knee. Pitfalls in the diagnosis and management. Eur J Orthop Surg Traumatol, 2001, 11: 255-257. [CrossRef]

5) Rompen JC, Ham SJ, Halbertsma JP, et al.: Gait and function in patients with a femoral endoprosthesis after tumor resection: 18 patients evaluated 12 years after surgery. Acta Orthop Scand, 2002, 73: 439-446. [Medline] [CrossRef]

6) Carty CP, Bennett MB, Dickinson IC, et al.: Assessment of kinematic and kinetic patterns following limb salvage procedures for bone sarcoma. Gait Posture, 2009, 30: 547-551. [Medline] [CrossRef]

7) Okita Y, Tatematsu N, Nagai K, et al.: Compensation by nonoperated joints in the lower limbs during walking after endoprosthetic knee replacement following bone tumor resection. Clin Biomech (Bristol, Avon), 2013, 28: 898-903. [Medline] [CrossRef]

8) Shehadeh A, El Dahleh M, Salem A, et al.: Standardization of rehabilitation after limb salvage surgery for sarcomas improves patients' outcome. Hematol Oncol Stem Cell Ther, 2013, 6: 105-111. [Medline] [CrossRef]

9) Katrak P, O'connor B, Woodgate I: Rehabilitation after total femur replacement: a report of 2 cases. Arch Phys Med Rehabil, 2003, 84: 1080-1084. [Medline] [CrossRef]

10) Lee A, Park J, Lee S: Gait analysis of elderly women after total knee arthroplasty. J Phys Ther Sci, 2015, $27: 591-595$. [Medline] [CrossRef]

11) De Visser E, Mulder T, Schreuder HW, et al.: Gait and electromyographic analysis of patients recovering after limbsaving surgery. Clin Biomech (Bristol, Avon), 2000, 15: 592-599. [Medline] [CrossRef]

12) Okita Y, Tatematsu N, Nagai K, et al.: Characteristics of flexed knee gait and functional outcome of a patient who underwent knee reconstruction with a hingeless prosthesis for bone tumor resection: a case report with gait analysis and comparison with healthy subjects. Eur J Phys Rehabil Med, 2013, 49: 849-855. [Medline]

13) de Visser E, Pauwels J, Duysens JE, et al.: Gait adaptations during walking under visual and cognitive constraints: a study of patients recovering from limb-saving surgery of the lower limb. Am J Phys Med Rehabil, 1998, 77: 503-509. [Medline] [CrossRef]

14) Colangeli M, Donati D, Benedetti MG, et al.: Total knee replacement versus osteochondral allograft in proximal tibia bone tumours. Int Orthop, 2007, 31: 823-829. [Medline] [CrossRef]

15) Lovecchio N, Zago M, Sciumè L, et al.: Squat exercise to estimate knee megaprosthesis rehabilitation: a pilot study. J Phys Ther Sci, 2015, 27: 2409-2412. [Medline] [CrossRef]

16) Heisel C, Breusch SJ, Schmid G, et al.: Lower limb salvage surgery with MUTARS endoprostheses: 2 to 7 year results. Acta Orthop Belg, 2004, 70: 142-147. [Medline]

17) Capanna R, Scoccianti G, Campanacci DA, et al.: Surgical technique: extraarticular knee resection with prosthesisproximal tibia-extensor apparatus allograft for tumors invading the knee. Clin Orthop Relat Res, 2011, 469: $2905-2914$. [Medline] [CrossRef]

18) Schraeder TL, Terek RM, Smith CC: Clinical evaluation of the knee. N Engl J Med, 2010, 363: e5. [Medline] [CrossRef]

19) Clarkson HM: Musculoskeletal Assessment: Joint Range of Motion and Manual Muscle Strength, 2nd ed. Philadelphia: Lippincott Williams \& Wilkins, 2000.

20) Reese NB: Muscle and Sensory Testing, 2nd ed. St Louis: Elsevier Saunders, 2005.

21) Zifchock RA, Davis I, Higginson J, et al.: The symmetry angle: a novel, robust method of quantifying asymmetry. Gait 
Posture, 2008, 27: 622-627. [Medline] [CrossRef]

22) McGill R, Tukey JW, Larsen WA: Variations of boxplots. Am Stat, 1978, 32: 2-16.

23) Biau D, Faure F, Katsahian S, et al.: Survival of total knee replacement with a megaprosthesis after bone tumor resection. J Bone Joint Surg Am, 2006, 88: 1285-1293. [Medline] [CrossRef]

24) Neumann DA: Kinesiology of the Musculoskeletal System, 2nd ed. London: Mosby, 2002.

25) Perry J, Burnfield JM: Gait analysis: normal and pathological function, 2nd ed. Thorofare: Slack Incorporated, 2010.

26) Baj A, Lovecchio N, Bolzoni A, et al.: Stair ascent and descent in assessing donor-site morbidity following osteocutaneous free fibula transfer: a preliminary study. J Oral Maxillofac Surg, 2015, 73: 184-193. [Medline] [CrossRef]

27) Chou SW, Liao HT, Yazar S, et al.: Assessment of fibula osteoseptocutaneous flap donor-site morbidity using balance and gait test. J Orthop Res, 2009, 27: 555-560. [Medline] [CrossRef]

28) Winter DA: Biomechanics and Motor Control of Human Movement, 1st ed. New York: Wiley, 1990.

29) Maruyama T, Sawada Y, Kubo S, et al.: Postoperative changes in knee joint function of total knee arthroplasty patients. J Phys Ther Sci, 2011, 23: 719-724. [CrossRef] 